\title{
Evaluation of Pathogenicity of Four Different Alternaria brassicae Isolates on Three Brassica coenospecies
}

\author{
Prashant Yadav ${ }^{1}$, Zahoor Ahmed Mir ${ }^{1}$, Sajad Ali ${ }^{2}$ and Anita Grover ${ }^{1 *}$ \\ ${ }^{1}$ ICAR - National Institute for Plant Biotechnology, New Delhi, India \\ ${ }^{2}$ Centre of Research for Development, University of Kashmir, Jammu and Kashmir, India \\ *Corresponding author
}

\begin{tabular}{|l|}
\hline Ke y w o r d s \\
$\begin{array}{l}\text { Alternaria } \\
\text { brassicae, Disease } \\
\text { resistance, Mustard, } \\
\text { Pathogenicity }\end{array}$ \\
\hline Article Info \\
\hline $\begin{array}{l}\text { Accepted: } \\
\text { 12 June } 2019 \\
\text { Available Online: } \\
\text { 10 July } 2019\end{array}$ \\
\hline
\end{tabular}

\section{A B S T R A C T}

\section{Introduction}

Among the different edible oilseed crops grown in the country (viz., soybean, rapeseedmustard, groundnut, sunflower, sesame, linseed, palm oil etc), Indian mustard ( $B$. juncea) is the second most important edible oilseed crop after soybean. B. juncea is a good source of healthy fatty acids like omega- 3 and omega- 6 fatty acids, and is very popular edible oil in northern states of the country. Presently, mustard is grown in area of 6.0 Mha with total production and productivity of 7.9 MMT and $1319 \mathrm{Kg} / \mathrm{ha}$ respectively (FAO. 2017). The production of edible oil in the country is lagging behind the consumption rate forcing 
India to import 14.5 MMT of edible oil in 2017-18 (The Solvent Extractors Association of India, 2018). Food and Agriculture Organization has estimated that India has highest edible oil consumption growth rate $(3.1 \%)$ in the world, that will lead the per capita consumption upto $24 \mathrm{Kg} /$ person by 2027 (FAO, 2018). The production of mustard can be enhanced by preventing the losses due to various biotic and abiotic stresses. Alternaria blight is a major biotic factor causing yield losses upto $36.8 \%$ in severe conditions (Bal and Kumar 2014).

Alternaria brassicae is the causal organism of Alternaria blight and it is a member of phylum Ascomycota. It is a necrotrophic fungal pathogen which kills its hosts by producing fungal toxins (Pedras and Smith, 1997). It produces brown-lack typical concentric ring spots popularly known as 'target board symptoms'. A. brassicae asexually produces large, multi-cellular and muriform conidia. The biochemical control (fungicides) of $A$. brassicae is available but its application leads to the increased cost of cultivation and environmental concerns.

Therefore, breeding for Alternaria resistant cultivars is a best strategy to prevent the losses through Alternaria blight. But in Brassica primary gene pool, the Alternaria resistant source is absent. However, some member of the Brassica coenospecies (viz Sinapis alba and Camelina sativa) possess resistance against A. brassicae (Sharma et al., 2002). S. alba and $C$. sativa are minor crops, currently grown in limited are for seeds and oil in Europe, USA, Canada and Australia. To develop the disease resistant cultivar, it is very important to test the improved genotype against virulent strain/isolates of the pathogen. For this, we studied the virulence of four different isolates of $A$. brassicae on the three Brassica coenospecies from 3 to 12 days post inoculation (dpi).

\section{Materials and Methods}

\section{Plant growth and infection}

Seeds of all the three species (available in the lab) were grown in plastic pots filled with soil and vermicompost at the nethouse of the ICAR - Indian agricultural research Institute, New Delhi during the winter season of 201819. A. brassicae isolates were collected from four different locations of the North India viz ICAR-IARI, New Delhi (isolate named as DEL), Govind Ballabh Pant University of Agriculture and Technology, Pantnagar, Uttarakhand (PNT), Sardar Vallabhbhai Patel University of Agriculture and Technology, Meerut (MRT), and Narendra Deva University of Agriculture and Technology, Ayodhya (ND). Mustard leaves infected with $A$. brassicae were collected from the fields of the above locations and pure culture was isolated on radish extract agar media. Spore suspension was prepared from the $A$. brassicae plates and concentration was adjusted to $5 \times 10^{3}$ spores $/ \mathrm{ml}$. Plants of the three Brassica coenospecies were infected with $A$. brassicae spores under control temperature $\left(25-30{ }^{\circ} \mathrm{C}\right)$ and humidity $(100 \%$ $\mathrm{RH})$ conditions in a poly-house.

\section{Disease progression}

A. brassicae infected plants of $B$. juncea, $S$. alba and $C$. sativa were observed for the disease progression from 3-12 days post inoculation. For studying the virulence of $A$. brassicae on the three Brassica coenospecies, parameters like lesion size, number of secondary spots and number of distal infected leaves were recorded at 0, 3, 6, 9 and 12 days post inoculation. Lesion size (in $\mathrm{cm}$ ) measured with the help of a 6 inch scale at time interval. The spots appearing alongside the primary inoculation as result of disease progression were considered as secondary spots. For disease scoring, a modified rating scale (0-6) 
was used based on the system given by Conn et al., (1990). Necrotic spots along with the surrounding yellowish necrotic area were considered as area under the pathogen attack. The non-inoculated leaves from the same plants were considered as distal leaves and distally infected leaves were considered as a parameter to study the virulence of each isolates of $A$. brassicae.

\section{Statistical analysis}

The experiment was conducted in a completely randomized design (CRD) and three replications were taken for each observation. Five leaves from each species for all the parameters were taken in one replication. Statistical analysis was done by using R software.

\section{Results and Discussion}

Indian mustard is an important source of edible oil in the country and its productivity is hampered by the Alternaria blight disease (Meena et al., 2016). To identify the high pathogenic strain/isolate of $A$. brassicae, four different isolates (PNT, ND, NRT and DEL) were studied on three Brassica coenospecies (B. juncea, S. alba and C. sativa). Disease parameters like lesion size, number of secondary spots and number distal infected leaves were studied from 3-12 days post inoculation. Lesion size was found highest in with PNT isolate $[1.23( \pm 0.24)]$ whereas lowest lesion size was observed with DEL isolate $[0.96( \pm 0.21)]$ in $B$. juncea leaves at 12 dpi. Lesion size in $S$. alba and $C$. sativa was observed highest with PNT infection whereas with DEL isolate, no disease spots were observed in C. sativa. Sharma et al., (2002) had observed similar results in case of $S$. alba and $C$. sativa against $A$. brassicae.

Secondary infection appeared as early as $6 \mathrm{dpi}$ in B. juncea with PNT $[8.7( \pm 1.7)]$ isolate of $A$. brassicae. In $B$. juncea, lowest number of secondary spots was observed with MRT $[7.0( \pm 2.2)]$ isolate. No secondary infection was observed in $C$. sativa with any of the isolates indication high resistance of $C$. sativa against $A$. brassicae.

For $S$. alba, highest and lowest number of secondary infection was observed with PNT $[2.7( \pm 0.6)]$ and DEL $[1.6( \pm 0.8)]$ isolates respectively. Mazumder et al., (2013) reported that the resistance in $S$. alba was due to the involvement of abscisic acid (ABA) mediated pathway and susceptibility in B. juncea is due to salicylic acid (SA) mediate defense pathway.

This is may be the reason for observing less number of secondary infections in $S$. alba in comparison to $B$. juncea.

Number of distal infected leaves was observed highest in $B$. juncea with PNT isolate $[5.1( \pm 0.19)]$ and lowest with DEL isolate $[3.8( \pm 0.2 .1)]$. In $S$. alba, highest number of distal infected leaves was reported with PNT $[2.3( \pm 0.94)]$ and lowest with DEL $[0.8( \pm 0.3 .2)]$ isolate at $12 \mathrm{dpi}$. C. sativa showed no distal infected leaf by any of the isolate. Based on the scoring of the $A$. brassicae infected leaves of the three Brassica coenospecies, $C$. sativa was observed highly resistant (R), S. alba moderately resistant (MR) and B. juncea highly susceptible (S) against this necrotrophic fungal pathogen.

Sharma et al., (2002) and Nayanakantha (2014) reported similar degree of resistance in these three Brassica coenospecies. Camelina sativa produces a phytoalexin called camalexin ((3-thiazol-20-yl-indole) which is known to detoxify fungal toxins (Purnamasari et al., 2015). Meena et al., (2017) studied the virulence of 30 different isolates of $A$. brassicae collected from various geographical locations of the country (Fig. 1 and 2; Table $1)$. 
Fig.1 Location of the four $A$. brassicae isolates shown on the map. (1=DEL, 2=MRT, 3=PNT and $4=\mathrm{ND}$ )

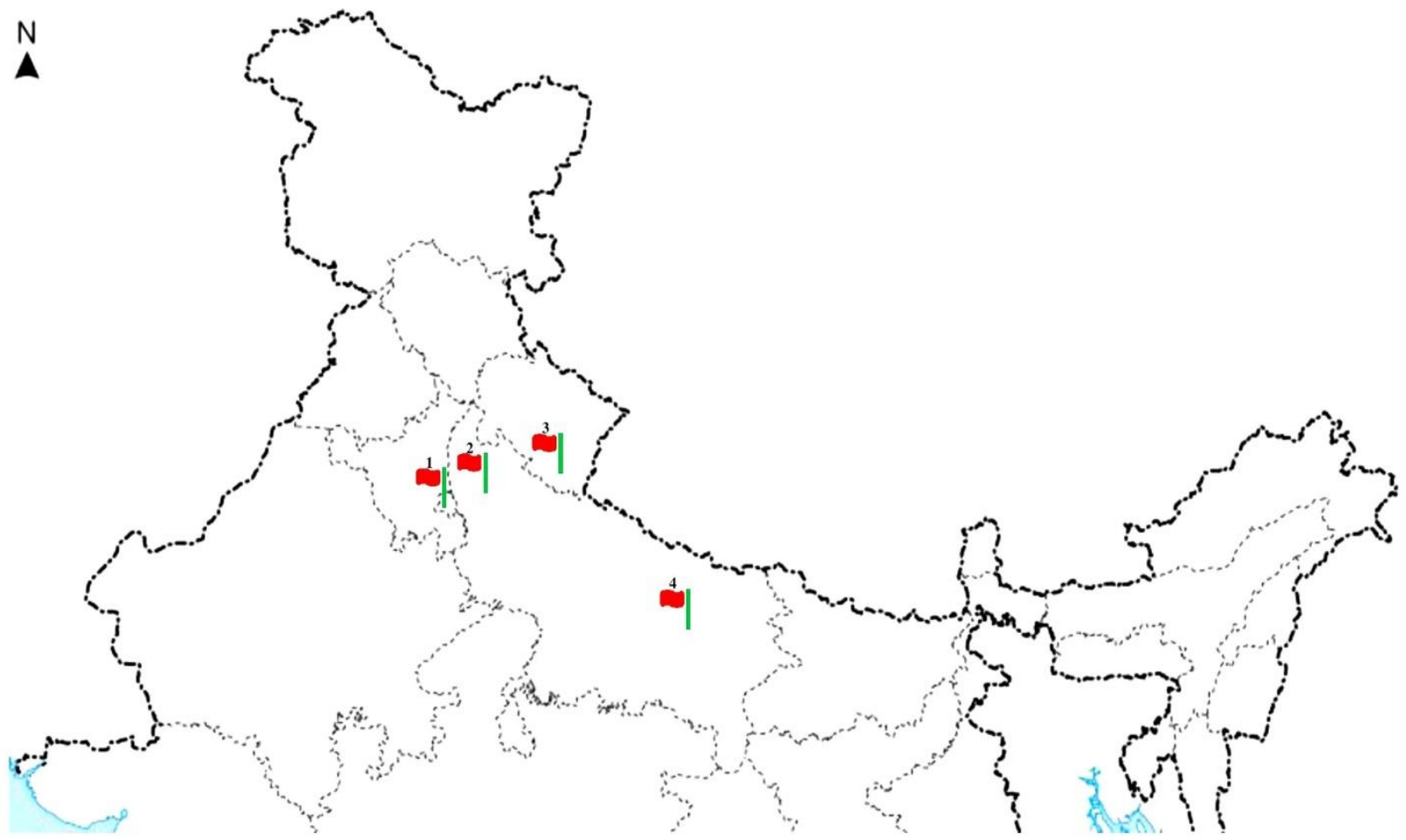

Fig.2 Control and A. brassicae inoculated leaves of $B$. juncea at 0 dpi and 3 dpi. Water was used in the control whereas $A$. brassicae spore suspension was used for the inoculation
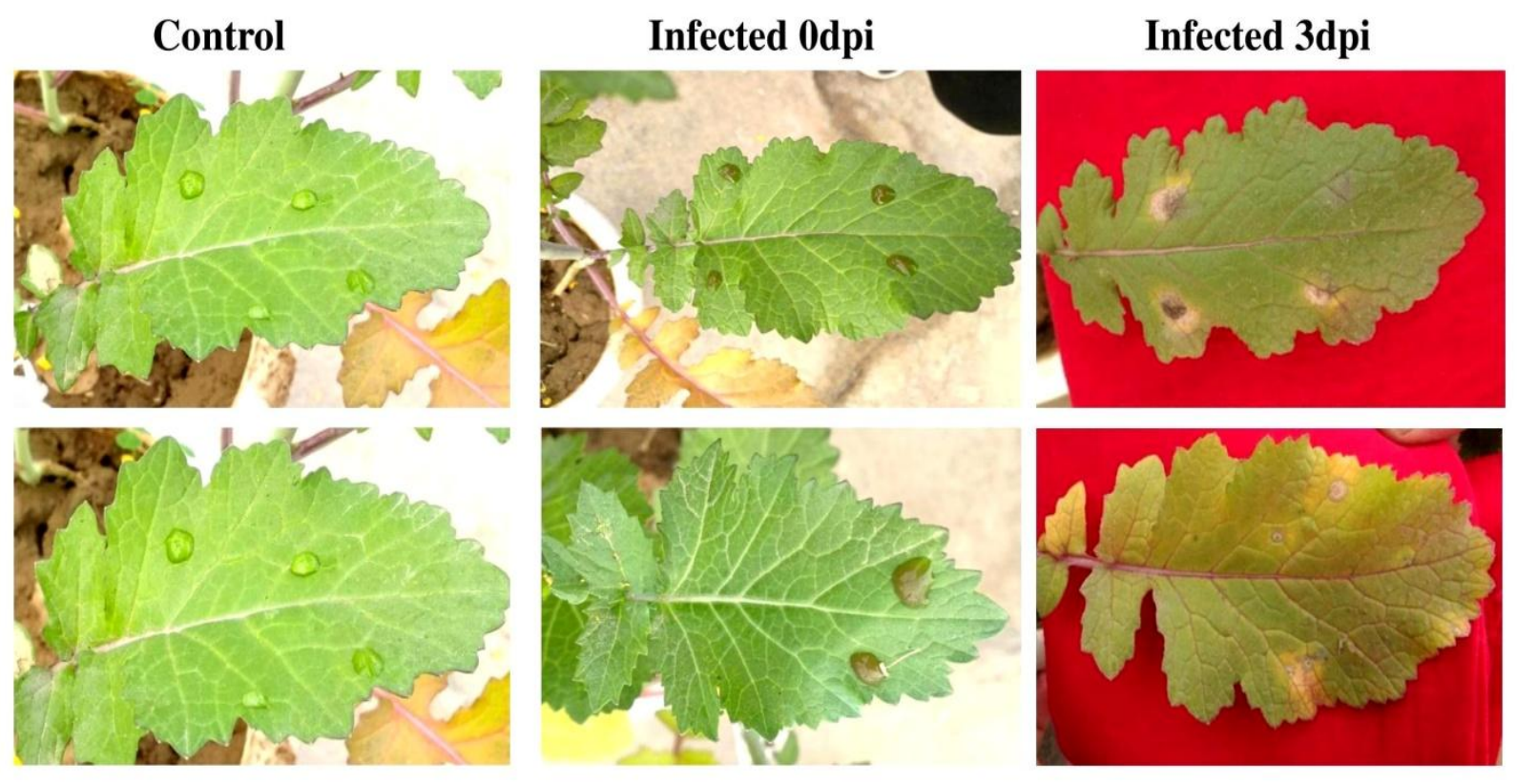
Table.1 Estimation of pathogenicity of A. brassicae isolates (PNT, ND, MRT and DEL) infected leaves of B. juncea, $S$. alba and $C$. sativa under control and at $12 \mathrm{dpi}$. Presented data showed mean \pm S.E. values estimated by taking 15 leaves from each species $(S=$ susceptible, $M R=$ moderately resistant, and $\mathrm{R}=$ resistant)

\begin{tabular}{|c|c|c|c|c|}
\hline Species & Mean lesion size (cm) & No of secondary spots & No of infected distal leaves & Rating \\
\hline \multicolumn{5}{|c|}{ Virulence of $A$. brassicae isolate (PNT) } \\
\hline B. juncea & $1.24( \pm 0.22)$ & $8.8( \pm 1.7)$ & $5.3( \pm 0.19)$ & S \\
\hline S. alba & $0.86( \pm 0.12)$ & $2.8( \pm 0.6)$ & $2.4( \pm 0.94)$ & MR \\
\hline C. sativa & $0.79( \pm 0.34)$ & $0.0( \pm 0.0)$ & $0.0( \pm 0.00)$ & $\mathrm{R}$ \\
\hline \multicolumn{5}{|c|}{ Virulence of $A$. brassicae isolate (ND) } \\
\hline B. juncea & $1.20( \pm 0.22)$ & $8.2( \pm 1.3)$ & $4.8( \pm 0.15)$ & $\mathrm{S}$ \\
\hline S. alba & $0.76( \pm 0.21)$ & $2.2( \pm 1.6)$ & $2.0( \pm 0.36)$ & MR \\
\hline C. sativa & $0.38( \pm 0.16)$ & $0.0( \pm 0.0)$ & $0.0( \pm 0.00)$ & $\mathrm{R}$ \\
\hline \multicolumn{5}{|c|}{ Virulence of $A$. brassicae isolate (MRT) } \\
\hline B. juncea & $1.02( \pm 0.14)$ & $7.0( \pm 2.2)$ & $4.5( \pm 0.12)$ & $\mathrm{S}$ \\
\hline S. alba & $0.81( \pm 0.22)$ & $2.1( \pm 1.2)$ & $1.8( \pm 0.24)$ & MR \\
\hline C. sativa & $0.32( \pm 0.14)$ & $0.0( \pm 0.0)$ & $0.0( \pm 0.00)$ & $\mathrm{R}$ \\
\hline \multicolumn{5}{|c|}{ Virulence of $A$. brassicae isolate (DEL) } \\
\hline B. juncea & $0.96( \pm 0.21)$ & $7.2( \pm 1.4)$ & $3.8( \pm 0.2 .1)$ & $S$ \\
\hline S. alba & $0.62( \pm 0.13)$ & $1.6( \pm 0.8)$ & $0.8( \pm 0.3 .2)$ & MR \\
\hline C. sativa & $0.0( \pm 0.00)$ & $0.0( \pm 0.0)$ & $0.0( \pm 0.00)$ & $\mathrm{R}$ \\
\hline
\end{tabular}

They observed that the isolates from the Pantnagar were grouped into virulent category whereas, isolated from the Assam, Meghalaya, Nagaland, Bharatpur and Kangra were grouped into highest virulent category. Our study is on isolates from three Northern states of India in contrast to Meena and coworker's work on isolates from different Indian states including North-East Indian states. Based on these parameters, isolate PNT was found highly virulent in B. juncea (as also reported by Meena et al., 2017) followed by ND and MRT isolates. DEL isolate was found least virulent in $B$. juncea among the four different isolates. Pramila et al., (2014) also found that the pathogenicity of A. brassicae isolate from Pantnagar was higher among different isolates studied from the Uttarakhand. Present study shows that there is variability in the virulence of different isolates of $A$. brassicae on the three Brassica coenospecies. Our research conclude that the highest pathogenic strain/isolate of $A$. brassicae was from the Pantnagar, Uttarakhand and it can be used for the future screening methods to develop A. brassicae resistant lines of Indian mustard.

\section{Acknowledgment}

Authors are thankful to the Project Director, National Institute for Plant Biotechnology, New Delhi, for providing research facilities and equipments.

\section{References}

Bal RS, Kumar A (2014) Studies on the epidemiology of white rust and Alternaria leaf blight and their effect on the yield of Indian mustard. Afr J Agric Res 9:302-306 
Conn, K. L., Tewari, J. P., and Awasthi, R. P. (1990). A disease assessment key for Alternaria blackspot in rapeseed and mustard. Disease des plantes Survey'au Canada, 70(1), 19.

FAO (2017). FAOSTAT. http://www.fao.org/faostat/en/\#data/QC/ visualize

FAO (2018). Oilseeds and oilseed products. OECD-FAO Agricultural Outlook 2018-2027.

http://www.fao.org/3/i9166e/i9166e_Ch apter4_Oilseeds.pdf

Mazumder, M., Das, S., Saha, U., Chatterjee, M., Bannerjee, K., and Basu, D. (2013). Salicylic acid-mediated establishment of the compatibility between Alternaria brassicicola and Brassica juncea is mitigated by abscisic acid in Sinapis alba. Plant physiology and biochemistry, 70, 43-51.

Meena, P. D., Gupta, R., Meena, H. S., Sharma, P., and Jambhulkar, S. (2017). Pathogenic variability within Indian Alternaria brassicae isolates using seed, cotyledon and leaf of Brassica species. Journal Phytopathology, 165(4), 238-248.

Meena, P. D., Jambhulkar, S. J., Gupta, R., Meena, H. S., and Singh, D. (2016). Rapid screening technique for Alternaria blight resistance in Indian mustard (Brassica juncea L.) using cotyledonary leaf method. Journal of Plant Pathology, 463-469.

Nayanakantha, N. M. C. (2014). Analysis of defense gene induction in Brassica juncea, Sinapis alba and Camelina sativa in response to Alternaria brassicae challenge and treatments with salicylic and jasmonic acids (Doctoral dissertation, National Research Centre On Plant Biotechnology Indian Agricultural Research Institute New Delhi).

Pedras M.S.C. and Smith K.C. (1997). Sinalexin, a phytoalexin from white mustard elicited by destruxin B and Alternaria brassicae. Phytochemistry 46: 833-837.

Pramila, P. G., Tasleem, M., Taj, G., Mal, R., and Kumar, A. (2014). Morphological, cultural, pathogenic and molecular variability amongst Indian mustard isolates of Alternaria brassicae in Uttarakhand. African Journal of Biotechnology, 13(3).

Purnamasari, M., Cawthray, G. R., Barbetti, M. J., Erskine, W., and Croser, J. S. (2015). Camalexin Production in Camelina sativa is Independent of Cotyledon Resistance to Sclerotinia sclerotiorum. Plant disease, 99(11), 1544-1549.

Sharma G., Dinesh Kumar V., Haque A., Bhat S.R., Prakash S. and Chopra V.L. (2002). Brassica coenospecies: a rich reservoir for genetic resistance to leaf spot caused by Alternaria brassicae. Euphytica 125: 411-417.

The Solvent Extractors Association of India (2018). Import of Vegetable Oil November 2017 to Oct 2018. https://seaofindia.com/import-ofvegetable-oil-november-2017-to-may2018/

\section{How to cite this article:}

Prashant Yadav, Zahoor Ahmed Mir, Sajad Ali and Anita Grover. 2019. Evaluation of Pathogenicity of Four Different Alternaria brassicae Isolates on Three Brassica coenospecies. Int.J.Curr.Microbiol.App.Sci. 8(07): 1426-1431. doi: https://doi.org/10.20546/ijcmas.2019.807.170 